\title{
Gentamicin and amikacin adversely affect male infertility indicated by pharmacological, andrological and pathological evidence
}

\author{
Hozaifa K. Elsawah ${ }^{1}$, Mohamed M. Kandiel' ${ }^{2}$, Aziza A. Amin ${ }^{3}$, \\ Haitham M. Mokhimar', AbuBakr M. El Mahmoudy ${ }^{1 *}$
}

${ }^{1}$ Department of Pharmacology, ${ }^{2}$ Department of Theriogenology, ${ }^{3}$ Department of Pathology, Benha University Faculty of Veterinary Medicine, Moshtohor, Qalyoubia, Egypt

Received: 20 November 2019

Accepted: 03 January 2020

*Correspondence:

Dr. AbuBakr M. El Mahmoudy,

Email: a.elmahmoudy@fvtm.bu.edu.eg

Copyright: (c) the author(s), publisher and licensee Medip Academy. This is an open-access article distributed under the terms of the Creative Commons Attribution Non-Commercial License, which permits unrestricted non-commercial use, distribution, and reproduction in any medium, provided the original work is properly cited.

\begin{abstract}
Background: Many drugs are implicated in male infertility and screening for medication history is an important for diagnosis and treatment of the problem. The aim is to study amikacin effect on male reproductive system in comparison to gentamicin.

Methods: Twenty-five male wister rats weighted $220 \pm 20 \mathrm{gm}$ and aged 8 weeks were randomly divided into five groups of five. The first group received gentamicin in dose $18.25 \mathrm{mg} / \mathrm{kg} /$ day once daily (OD) (therapeutic dose). The second group received gentamicin with double dose of the first group. The third group received amikacin in dose $54.75 \mathrm{mg} / \mathrm{kg} /$ day OD (therapeutic dose). The Fourth group received amikacin with double dose of the third group. However, the fifth group served as a control and received normal saline (NS) OD. All treatments were administered intraperitoneally (IP) for 14 days. On the 15th day, blood samples and reproductive organs were obtained from all animals. Testicular tissues were prepared for genetic testing and chemical and microscopical examination.

Results: Amikacin and gentamicin negatively affected reproductive organs weights, sperm parameters, serum follicle stimulating hormone and luteinizing hormone $(\mathrm{LH})$ level relative to control $(\mathrm{p}<0.05)$. However, serum testosterone level was only affected with gentamicin $(\mathrm{p}<0.05)$. A significant difference between gentamicin and amikacin was found in sperm count, testis and epididymis weights and serum testosterone and LH level $(p<0.05)$. Testicular histopathological changes were also found with the two drugs with different degrees. Effects of both gentamicin and amikacin were dose-dependent.

Conclusions: Both gentamicin and amikacin adversely affect andrological function that should be monitored and controlled during application of these drugs.
\end{abstract}

Keywords: Gentamicin, Amikacin, Hormone, Testis, Sperm, Reproduction

\section{INTRODUCTION}

Aminoglycoside antibiotics are one of the main bactericidal antibiotic classes commonly used for different types of infection e.g. urinary tract infection, infective endocarditis and blood stream infection. ${ }^{1-3}$ Aminoglycosides are potent, broad-spectrum antibiotics that act through inhibition of protein synthesis. ${ }^{4}$ The aminoglycosides have been a cornerstone of antibacterial chemotherapy since streptomycin was first isolated from Streptomyces griseus and introduced into clinical use in 1944, other members include: kanamycin, neomycin, gentamicin, netilmicin, tobramycin, amikacin, and newer members: arbekacin and plazomicin. ${ }^{5}$

Gentamicin and amikacin are similar in spectrum of activity with some antipseudomonal advantages to 
amikacin, and similar in nephrotoxicity with less nephrotoxic effect to amikacin. 6,7

It was established through many studies that gentamicin produces oxidative stress in different cell types of the body accompanied with direct gonadotoxic effect. ${ }^{8,9}$ However, based on available information, direct comparison between gentamicin and amikacin regarding negative effect on male reproductive system and their effect on oxidative stress has not been studied. Therefore, the aim of the present study is to disclose the possible effect of amikacin on male reproductive system of male albino rat though investigating reproductive organs weights, sperm parameters, testicular histopathology and serum testosterone and gonadotropin hormones in comparison to gentamicin.

\section{METHODS}

\section{Drug preparation}

Gentamicin was purchased as a brand pharmaceutical preparation Garamycin ${ }^{\circledR}$ with concentration $80 \mathrm{mg}$ of gentamicin in $2 \mathrm{ml}$ aqueous solvent manufactured by Memphis Co. for pharmaceutical and chemical industry (MEMCO) under authority of Schering-Plough corporation U.S.A. Gentamicin was diluted with normal saline (NS) to a final volume $0.5 \mathrm{ml}$ per rat to be injected intraperitoneal (IP) with 25-gauge needle. Amikacin was purchased as a brand pharmaceutical preparation Amikin ${ }^{\circledR}$ with concentration $500 \mathrm{mg}$ of amikacin in $2 \mathrm{ml}$ aqueous solvent manufactured by SmithKline Beecham Egypt L.L.C.; an affiliated Co. to GlaxoSmithKline (GSK), USA. Amikacin was diluted with NS to a final volume $0.5 \mathrm{ml}$ per rat to be injected IP with 25 -gauge needle.

\section{Animals}

Twenty-five adult wister albino male rats were 8 weeks old and weighted $220 \pm 20 \mathrm{~g}$. They were obtained from animal house of faculty of veterinary medicine, Benha university. Male rats were housed in normal room temperature with humidity (40-60\%) and $12 \mathrm{~h} / 12 \mathrm{~h}$ light or dark cycle prior to use in experimental protocols. The animals were fed laboratory formula and tap water ad libitum.

\section{Study design}

After two weeks of adaptation to a standard diet, the 25 rats were randomly divided into five groups with five rats in each group. Rats in group 1 and 2 received IP gentamicin once daily (OD) in daily doses of 18.25 and $36.5 \mathrm{mg} / \mathrm{kg}$, respectively. Rats in group 3 and 4 received IP amikacin OD in daily doses of 54.75 and $109.5 \mathrm{mg} / \mathrm{kg}$, respectively. Group 5 served as control and received IP $0.5 \mathrm{ml} \mathrm{NS}$ OD. All treatments were administered for 14 days. On the fifteenth day, the five animals in each group were sacrificed by cervical dislocation under anaesthesia after blood sampling. Immediately after dissection, testis, epididymis, prostate, and seminal vesicle were removed and weighted (absolute and relative to body weight). Relative organ weight was expressed as organ weight (mg) or body weight $(\mathrm{g})$. The experiment was carried out in the departments of pharmacology and theriogenology, faculty of veterinary medicine, Benha university.

\section{Blood sample collection}

Blood samples were collected from the retro-orbital venous plexus located at the medial canthus of the eye by means of heparinized capillary tubes. The collected blood was kept in heparinized test tubes and centrifuged at 1500 $\mathrm{xg}$ for 15 minutes then collected in sterile Eppendorf tubes using automatic pipettes. Plasma samples were kept frozen $\left(-20^{\circ} \mathrm{C}\right)$ till analysis of the following biochemical parameters: testosterone, follicle stimulating hormone (FSH) and luteinizing hormone (LH) using rat ELISA kits (Elabscience ${ }^{\circledR}$, Taxus, USA).

\section{Sperm parameter assessment}

Sperms were obtained from the tail of the two epididymis by cutting the epididymis into small pieces in petri dish containing $2 \mathrm{ml}$ of saline $(0.9 \% \mathrm{NaCl})$. For evaluation of sperm motility, immediately after collection of a semen sample, a small drop was taken with a micropipette and placed on a warm clean glass slide. A cover slip was placed onto the semen drop and the percentage of progressively motile sperms was estimated microscopically at 400X magnification. For evaluation of sperm morphology and viability, two types of seminal smear slides were obtained from each animal. One of them was stained with Eosin and Nigrosine and the other was fixed in Carnoy's solution then examined with light microscope under magnifications of 400X and 1000X to determine sperm head and tail abnormalities and sperm viability. For evaluation of sperm count, the epididymal contents extracted in $2 \mathrm{ml} \mathrm{NS}$ were diluted to $10 \mathrm{ml} \mathrm{NS}$ and kept for 24 hours to let sperms distribute in the solution. To stain sperms heads to be easily counted, a few drops of eosin 2\% solution were added to the solution before examination. According to the method described previously, the number of sperm in five large squares was multiplied by 5 to obtain sperm count in one chamber that contains 25 large squares (one chamber volume $=100 \mathrm{ml}) .{ }^{10}$ The number was multiplied by 10000 to obtain sperm count in $1 \mathrm{ml}$. Because we diluted semen in $10 \mathrm{ml}$ and used two epididymis, the number was multiplied by 10 and divided by 2 as the following:

Number of sperms per $1 \mathrm{ml}=$

No. of sperms in 5 large squares $\times 5 \times 100000$ 2 


\section{Histopathology of the testis}

The left testis was fixed in $10 \%$ formalin and embedded in paraffin. Five-micron thick sections were prepared and stained with hematoxylin and eosin (H\&E). The specimens were examined under Olympus or $3 \mathrm{H}$ light microscope under power of $200 \mathrm{X}$ and $400 \mathrm{X}$. the examination was carried out in the department of pathology.

\section{Statistical analysis}

Statistical analysis was performed by using GraphPad Prism 8.0.2 software. Numerical data in this study were expressed as mean \pm standard deviation $(\mathrm{M} \pm \mathrm{SE})$ and categorical data as percentages. Analysis of variance (ANOVA) and a post hoc Tukey test were used. The difference between groups was defined to be statistically significant when a $p$ value $\leq 0.05$.

\section{Ethics statement}

This study was carried out in strict accordance with the recommendations in the guide for the care and use of laboratory animals of the National Institutes of Health 8th edition and adhered to principles published by International Council for Laboratory Animal Science and those of Benha University Animal Care and Use Committee.

\section{RESULTS}

\section{Body and reproductive organ weights}

After 14 days of treatment, weights of the total body and ventral prostate didn't differ significantly among groups ( $p>0.05$ ). Weights of epididymis and seminal vesicle were significantly lower in animals treated with gentamicin and double dose amikacin relative to control $(\mathrm{p}<0.05)$. However, testis weight was decreased with all doses of the two drugs $(\mathrm{p}<0.05)$. A significant dose dependent manner was found in testis wt. (gentamicin) $(p<0.05)$ and seminal vesicle wt. (amikacin) $(\mathrm{p}<0.05)$. There were significant differences between gentamicin and amikacin in epididymis wt. (therapeutic dose) and testis wt. (two doses) $(\mathrm{p}<0.05)$ (Table 1).

\section{Sperm parameters}

Gentamicin and amikacin significantly decreased sperm count, progressive motility and tail normality relative to control $(p<0.05)$. However, the double dose of the two drugs significantly reduced sperm viability $(\mathrm{p}<0.05)$ and the double dose of amikacin significantly increased head abnormalities compared to control $(\mathrm{p}<0.05)$. A significant dose dependent effect was observed with progressive motility in all treated-groups $(\mathrm{p}<0.05)$ and viability in gentamicin-treated groups $(\mathrm{p}<0.05)$. A significant difference between the two treatments was seen with sperm count at the two dose levels $(\mathrm{p}<0.05)$ (Table 2$)$.

Table 1: Total body weight, absolute and relative reproductive organ weights of male rats after administration of gentamicin and amikacin with therapeutic and double dose for 14 days compared with control.

\begin{tabular}{|llllll|} 
& Control & \multicolumn{2}{l|}{ Gentamicin (mg/kg) } & \multicolumn{2}{l|}{ Amikacin (mg/kg) } \\
\hline Weight & & 18.25 & 36.5 & 54.75 & 109.5 \\
\hline Total body weight $(\mathbf{g})$ & $274 \pm 2.68$ & $271.2 \pm 4.3$ & $268.6 \pm 4.5$ & $264.0 \pm 2.3$ & $273.2 \pm 2.5$ \\
\hline Testis $(\mathbf{g})$ & $1.52 \pm .03$ & $1.134 \pm .02^{*}$ & $.902 \pm .03^{*}$ & $1.3 \pm .04^{*}$ & $1.2 \pm .05^{*}$ \\
\hline Epididymis (mg) & $556 \pm 16$ & $432 \pm 12^{*}$ & $384 \pm 11^{*}$ & $508 \pm 31$ & $445 \pm 20^{*}$ \\
\hline Seminal vesicle (mg) & $572 \pm 14$ & $448 \pm 14.5^{*}$ & $394 \pm 15^{*}$ & $530 \pm 26.5$ & $437 \pm 39^{*}$ \\
\hline Ventral prostate (mg) & $486 \pm 18$ & $448 \pm 7$ & $520 \pm 13$ & $496 \pm 24$ & $454 \pm 10.3$ \\
\hline Relative (R) testis (mg) & $554 \pm 11.5$ & $418.7 \pm 13^{*}$ & $336.3 \pm 14.5^{*}$ & $493 \pm 22$ & $441 \pm 21^{*}$ \\
\hline R epididymis (mg) & $202.2 \pm 4.5$ & $159.6 \pm 6.5^{*}$ & $143.2 \pm 6^{*}$ & $192.5 \pm 12.3$ & $163 \pm 7.5^{*}$ \\
\hline R seminal vesicle (mg) & $208.7 \pm 7.3$ & $161.3 \pm 4.4^{*}$ & $148.1 \pm 6^{*}$ & $196 \pm 8.2$ & $161 \pm 15.1^{*}$ \\
\hline R ventral prostate (mg) & $177.13 \pm 8.3$ & $165.4 \pm 4.5$ & $193.6 \pm 4.3$ & $187.7 \pm 7.5$ & $166.2 \pm 4$ \\
\hline
\end{tabular}

Values expressed as mean \pm SE of 5 observations, *: significant difference at $p<0.05$ compared with control. One-way ANOVA and post hoc Tukey test were used.

Table 2: Sperms count, motility, viability and morphology of male rats after administration of gentamicin and amikacin with therapeutic and double dose for 14 days compared with control.

\begin{tabular}{|llllll|}
\hline & Control & \multicolumn{2}{l}{ Gentamicin $(\mathbf{m g} / \mathrm{kg})$} & \multicolumn{3}{l|}{ Amikacin (mg/kg) } \\
\hline Sperm parameters & & 18.25 & 36.5 & 54.75 & 109.5 \\
\hline Progressive motility \% & $73 \pm 4.2$ & $41 \pm 3.6^{*}$ & $29 \pm 3.7^{*}$ & $48 \pm 4.5^{*}$ & $24.45 \pm 1.43^{*}$ \\
\hline Viability \% & $75 \pm 1.7$ & $71 \pm 2.1$ & $61 \pm 3.6^{*}$ & $69 \pm 2.1$ & $61.4 \pm 2.3^{*}$ \\
\hline Head abnormality \% & $3 \pm 0.6$ & $7.8 \pm 2.05$ & $15.8 \pm 3.4$ & $12 \pm 3.8$ & $24.6 \pm 5.2^{*}$ \\
\hline Tail abnormality \% & $20.6 \pm 2.5$ & $70.2 \pm 2.2^{*}$ & $68.4 \pm 3.6^{*}$ & $64.4 \pm 3.5^{*}$ & $57.4 \pm 3.1^{*}$ \\
\hline
\end{tabular}

Values expressed as mean \pm SE of 5 observations, *: significant difference at $\mathrm{p}<0.05$ compared with control. One-way ANOVA and post hoc Tukey test were used. 
Table 3: Plasma hormones level of male rats after administration of gentamicin and amikacin with therapeutic and double dose for 14 days compared with control.

\begin{tabular}{|llllll|}
\hline & Control & \multicolumn{2}{l}{ Gentamicin $(\mathrm{mg} / \mathrm{kg})$} & \multicolumn{2}{l|}{ A milkacin $(\mathrm{mg} / \mathrm{kg})$} \\
\hline Testosterone $(\mathbf{n g} / \mathbf{m l})$ & & 18.25 & 36.5 & 54.75 & 109.5 \\
\hline FSH $(\mathbf{n g} / \mathbf{m l})$ & $0.954 \pm 0.06$ & $0.424 \pm 0.02^{*}$ & $0.37 \pm 0.02^{*}$ & $0.874 \pm 0.06$ & $0.732 \pm 0.08$ \\
\hline LH $(\mathbf{m I U} / \mathbf{m l})$ & $1.232 \pm 0.05$ & $3 . .19 \pm 0.2^{*}$ & $3.28 \pm 0.3^{*}$ & $3.75 \pm 0.15^{*}$ & $3 \pm 0.3^{*}$ \\
\hline
\end{tabular}

Values expressed as mean \pm SE of 5 observations, *: significant difference at $\mathrm{p}<0.05$ compared with control. One-way ANOVA and post hoc Tukey test were used.
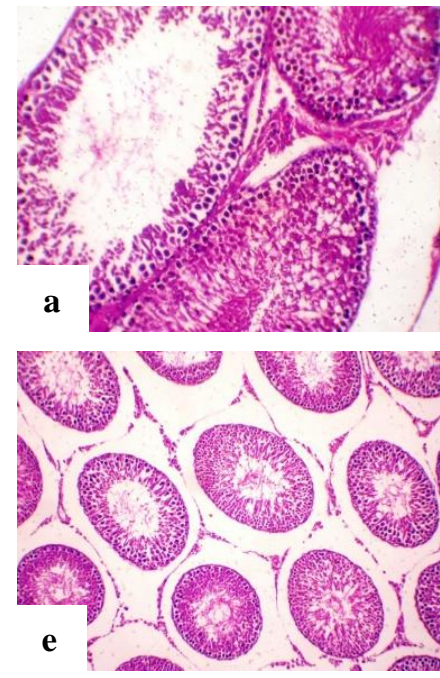
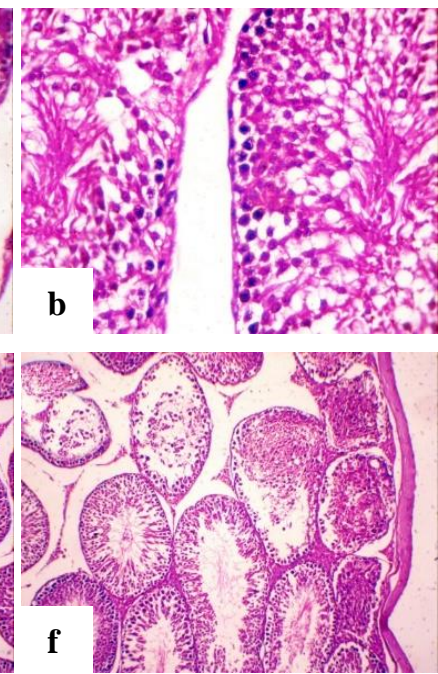
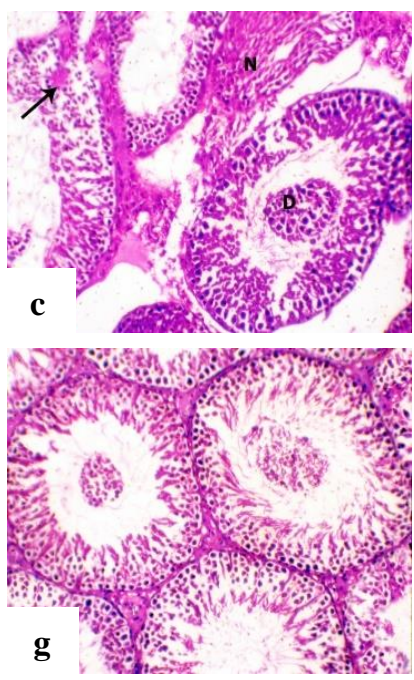

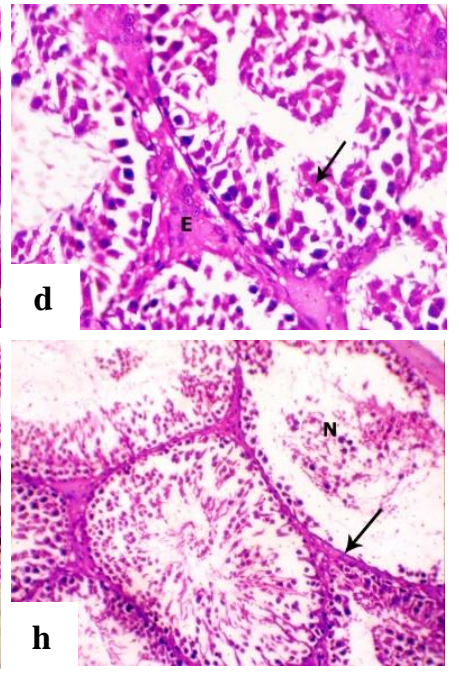

Figure 1: H\&E stained section of testes obtained from rats post administration of therapeutic dose gentamicin (a-d) and double dose gentamicin (e-h) for 2 weeks, showing (a) reduction in the number of spermatogonial cells lined seminiferous tubules (X400), (b) seminiferous tubules lined by degenerated germ cells with cytoplasmic vacuolation (X400), (c) degeneration (arrow) and entire necrosis of spermatogonial cells ( $N$ ) of some seminiferous tubules with impaction of the lumen of degenerated tubules with dead sperm $(\mathrm{D}, \mathrm{X400})$, (d) necrosis of the lining epithelium of seminiferous tubules and exfoliation of epithelial cells in their lumen (arrow) with mild inter-tubular edema (E,

X400), (e) atrophy of seminiferous tubules (X100), (f) variable degree of degenerative changes of germinal epithelial cells (X100), (g) disrupted tubular epithelium with testicular degeneration of most seminiferous tubules as well as impaction of their lumens with dead sperm (X200), (h) entire necrosis of germinal epithelium (arrow) with inhibition of spermatogenesis and Distension of the lumen with necrotic cells (N, X200).

\section{Testosterone, FSH and $\mathrm{LH}$}

Serum testosterone level was significantly reduced in all gentamicin- treated groups $(\mathrm{p}<0.05)$ and serum FSH and LH were significantly increased in all gentamicin and amikacin-treated groups relative to control $(\mathrm{p}<0.05)$. No significant dose dependent effect was seen $(p>0.05)$. The difference between the two antibiotics was significant in serum testosterone at the two doses level $(\mathrm{p}<0.05)$ (Table 3).

\section{Histopathological findings}

The histopathological examination of the testes obtained from rats treated with therapeutic dose gentamicin for 2 weeks revealed congestion of sub-capsular blood vessels with variable degree of degenerative changes of the lining epithelial cells of some seminiferous tubules in combination with incomplete spermatogenesis and absence of spermatozoa in the lumen of these degenerated tubules. Clear reduction in the number of spermatogenic cells and the sperm bundles were absent in some tubules (Figure 1a). Swollen, pale and vacuolated cytoplasm of the lining epithelium of some seminiferous tubules with accumulation of necrotic eosinophilic debris in the lumen of some seminiferous tubules (Figure 1b). Necrosis of spermatogenic cells of some seminiferous tubules with distension of their lumen with dead sperm in association with stop of spermatogenesis was also demonstrated in some examined cases (Figure 1c and d).

These histopathological changes were more marked in rats treated with double dose gentamicin for 2 weeks. The testes of these animals showed congestion of the testicular blood vessels and interstitial capillaries. The seminiferous tubules were more affected as marked atrophy of seminiferous tubules characterized by reduction in size of the tubules with increase in the distance between the tubules with extensive testicular degeneration of great 
numbers of seminiferous tubules was noticed (Figure 1e). The degenerated tubules exhibited variable degree of degenerative changes that characterized by marked reduction of germinal epithelial cells, accompanied by reduced spermatogenesis and absence of spermatozoa in the lumen (Figure 1f). Frequently, desquamated epithelial cells with dead spermatogenic cells were seen in the lumen of the degenerated tubules (Figure 1g).
Additionally, marked necrosis of spermatogenic cells in seminiferous tubules that lined by few layers of degenerated germ cells was observed and distension of the lumen of some tubules with necrotic cells and the sperm bundles were absent in most tubules (Figure 1h). Meanwhile, multiple areas of lytic necrosis of the germinal epithelium of seminiferous tubules that characterized by complete loss of spermatogenic cells was demonstrated.
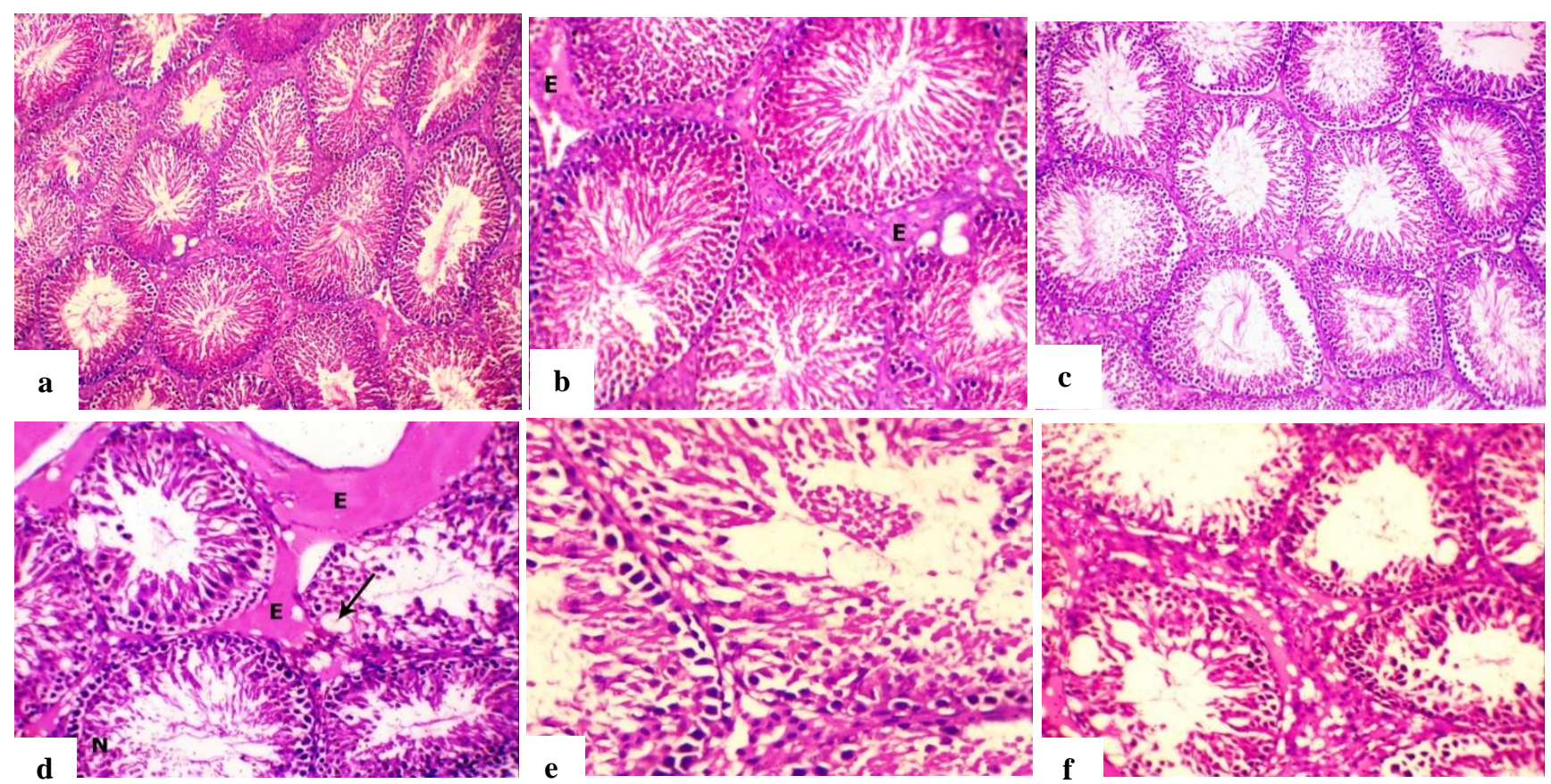

Figure 2: H\&E stained section of testes obtained from rats post administration of therapeutic dose amikacin (a-b) and double dose amikacin (e and f) for 2 weeks, showing (a) normal seminiferous tubules with normal spermatogenesis (X100), (b), mild vacuolation of the germinal epithelium of few seminiferous tubules with mild inter-tubular edema (E, X200), (c), degeneration of germinal epithelium with incomplete of spermatogenesis (X100), (d) severe degenerative changes with vacuolated epithelium (arrow) and marked inter-tubular edema (E, X200), (e) exfoliated epithelial cells in the lumen of degenerated tubule (X400), (f) degeneration of the lining epithelial cells of a seminiferous tubule characterized by swollen pale discrete large vacuoles, notice also, some seminiferous tubules lined by few layers of degenerated germ cells with absence of spermatozoa in the lumen (X400).

However, the microscopical examination of testes of animals treated for 2 weeks with therapeutic dose of amikacin revealed less prominent histopathological changes when compared with the group treated with gentamicin for the same period. Most of the seminiferous tubules were compact with each other and the spermatogenic layers appeared somewhat normal in most examined animals (Figure 2a), consequently, most of the seminiferous tubules restored its normal histological architecture except mild degeneration of the lining epithelium cells of some seminiferous tubules with mild inter-tubular edema (Figure 2b) was demonstrated. On the other side, the testes treated with double dose amikacin revealed more advanced pathological changes in comparison to the testes treated with therapeutic dose amikacin. Meanwhile, these pathological alterations were less prominent when compared with the testes of animals treated with therapeutic dose gentamicin. Degeneration of germinal epithelium and loss of spermatids with incomplete of spermatogenesis was observed in most examined cases (Figure 2c). Most of the spermatogonia showed degeneration with cytoplasmic vacuolization and marked inter-tubular edema (Figure 2d). Additionally, necrosis of some seminiferous tubular epithelium, with defoliation of many spermatocytes into the lumen of seminiferous tubules with incomplete spermatogenesis was also demonstrated (Figure 2e and f).

On the other side, normal histological structure of tunica albuginea, seminiferous tubules and interstitial tissue was detected during the histopathological examination of the testes taken from rats injected with NS (control).

\section{DISCUSSION}

Many drugs are implicated in male infertility and identification of these medications is very essential in the 
investigation of male infertility. ${ }^{11,12}$ The effects of gentamicin an amikacin on male reproductive system of wistar rats were investigated using the average doses of $\mathrm{Kg}$-based dosing that equal 5 and $15 \mathrm{mg} / \mathrm{kg} /$ day in gentamicin and amikacin respectively and body surface area-based dosing that equal 31.5 and $94.5 \mathrm{mg} / \mathrm{kg} / \mathrm{day}$ in gentamicin and amikacin respectively. ${ }^{13}$ So, the therapeutic doses are 18.25 and $54.75 \mathrm{mg} / \mathrm{kg} /$ day and the double doses are 36.5 and $109.5 \mathrm{mg} / \mathrm{kg} /$ day in gentamicin and amikacin respectively that fall in the previous dosing range. Gentamicin was used in Wister rat for reproductive studies with doses ranged from $3 \mathrm{mg} / \mathrm{kg} / \mathrm{day}$ to 80 $\mathrm{mg} / \mathrm{kg} /$ day and $100 \mathrm{mg} / \mathrm{kg} /$ day for durations ranged from 1 day to 14 days. ${ }^{9,14-16}$ To our knowledge, amikacin was used in only one infertility study in male rats with doses ranged from 25 to $200 \mathrm{mg} / \mathrm{kg} / \mathrm{day}$ for 60 days. ${ }^{17} \mathrm{~A}$ significant dose dependent effect of both gentamicin and amikacin on testis, epididymis and seminal vesicle weights was revealed by the present study. Gentamicin in a recent study reduced absolute testis weight $(\mathrm{p}<0.05)$ with doses 60,80 and $100 \mathrm{mg} / \mathrm{kg}$ OD for 10 days in a dose depend manner and relative testis weight with the higher two doses $(\mathrm{p}<0.05){ }^{18}$ Similar findings were reported with various gentamicin regimens; testis, epididymis and seminal vesicle weigh were reduced in 5 $\mathrm{mg} / \mathrm{kg} /$ day gentamicin-treated groups $\quad(\mathrm{p}<0.05) .{ }^{16}$ However, $3 \mathrm{mg} / \mathrm{kg} /$ day gentamicin didn't significantly reduce testis and epididymis weight $(p>0.05)$ that may be related to the low dose used. Another study used 100 $\mathrm{mg} / \mathrm{kg} /$ day for 6 days didn't find a significant effect on testis and seminal vesicle weight $(p>0.05)$ that may be because of the short duration of the treatment. ${ }^{9,19}$ The testis weight relies on seminiferous tubule and interstitial cells; therefore the reduction in germ cells decreases testicular weight. $^{20}$ Consistent with this, we found a histopathological evidence of testicular cells loss including germ cells. The present study revealed that testosterone was significantly reduced in gentamicintreated groups $(\mathrm{p}<0.05)$. In addition, FSH and LH were significantly increased in the all treated groups $(\mathrm{p}<0.05)$. However, testosterone wasn't significantly changed in amikacin-treated groups. Testosterone hormone was investigated in few studies with gentamicin treatment and revealed similar findings. A dose dependent reduction in testosterone level with gentamicin $60,80,100 \mathrm{mg} / \mathrm{kg} /$ day for 10 days was found and 4 days treatment with 40 $\mathrm{mg} / \mathrm{kg} /$ day gentamicin and 14 days treatment with dose 5 $\mathrm{mg} / \mathrm{kg} /$ day gentamicin reduced testosterone significantly. ${ }^{14,18,21}$ None of these studies measured FSH and TSH levels. However, FSH serum level was studied in a protective experimental study with gentamicin treatment and found that testosterone was reduced but FSH wasn't significantly affected. ${ }^{22}$ The rise in gonadotropins may be secondary to reduced feedback inhibition of testosterone. ${ }^{23}$ Testosterone is an essential hormone for the attachment of germ cells in the seminiferous tubules and prevent their apoptosis. ${ }^{24}$ Testosterone is important in maintaining testis weight through maintenance of the spermatogenic process and inhibition of germ cell apoptosis; therefore, testosterone reduction is associated with germ cell loss. However, testosterone is synthesized by the Leydig cells in the interstitial compartment of the testis and loss of Leydig cells results in decreased testosterone level. ${ }^{25}$ Wistar rats at 50 days age get the maximum number of Leydig cells per testis and have full spermatogenesis. However, testosterone was measurable in the testis at 20 days of age and reaches peak plasma level at 60 days of age to be $0.77 \pm 0.16$ and $4 \pm 0.73 \mathrm{mcg} / \mathrm{dl}$ in peripheral and spermatic vein respectively. ${ }^{26}$ In the present study, the sperm parameters were altered in gentamicin-treated group and to a lesser extent, amikacin. Consistent with this, gentamicin had a negative effect on sperm parameters including sperm motility, viability, count and morphology.9,16,18,19 The reduction in sperm count and maturation in gentamicin-treated group may be related to low testosterone level, increased testicular oxidative stress or both. ${ }^{15,25}$ Meanwhile, we found a correlation between sperm parameters, testosterone level and organs weight in gentamicin and amikacin treated groups. However, amikacin had lower effect on all correlated factors. Both sperm count and sperm motility are important in cervical penetration and ova fertilization. ${ }^{25}$ Histopathological changes of the testis with gentamicin treatment observed here are quite similar to findings reported by some previous studies. ${ }^{9,18,21,27}$ They found variable degrees of degenerative changes of the lining epithelial cells of some seminiferous tubules in combination with incomplete spermatogenesis and absence of spermatozoa as well as evidence of cell debris in the lumen and presence of lymphocyte and plasmocyte. However, amikacin in the present study revealed less prominent histopathological changes relative to gentamicin. Because germ cells are very rich in polyunsaturated fatty acid for energy production and cell membrane structure and because these molecules are vulnerable to oxidative damage, testis and epididymis germ cells are enhanced with potent scavenger systems. ${ }^{28}$ One possible mechanism of gentamicin and amikacin-induced testicular cell damage is oxidative stress where there was a significant increase in the levels of Hydrogen peroxide (H2O2) and lipid peroxides with decrease in activities of the antioxidant enzymes SOD, catalase and GPx and decreased glutathione content in the testes of gentamicin-treated group. ${ }^{18}$ Comparative effect of gentamicin and amikacin on testicular oxidative stress is needed to be studied that may give a possible explanation for the difference between the two drugs effect on male reproductive system. However, current investigations are being conducted by our team to explore drug-induced oxidative stress at cellular and molecular level. Another possible explanation is the blood-testis barrier; a tight junction between Sertoli cells that protect germ cells from substances penetration and there is a good correlation between lipophilicity of the drug and its permeability through blood-tissue barriers. ${ }^{29,30}$ Amikacin binds more loosely to phospholipid bilayers and is less taken up by tubular cells in vivo relative to gentamicin. ${ }^{31}$ Finally, human follow up studies are required to keep caution regarding negative effect of gentamicin and 
amikacin on human fertility specially couples seeking for children.

\section{CONCLUSION}

Treatment with either gentamicin or amikacin exhibited variable degrees of testicular degeneration via induction of histopathological alterations in the seminiferous tubules and interstitial tissues, gonadotropins and testosterone level and impaired sperm parameters. The severity of these changes was dose dependent. Amikacin relative to gentamicin is less gonadotoxic, but relative to control, amikacin retained some gonadotoxicity. Monitoring of patients on these drugs is recommended.

\section{ACKNOWLEDGEMENTS}

We gratefully thank doctors and other workers of the central laboratory of the faculty for their support and help specially Dr. Ibrahim Abdel-Wadoud for carrying out biochemical tests.

\section{Funding: No funding sources \\ Conflict of interest: None declared \\ Ethical approval: Not required}

\section{REFERENCES}

1. Gupta K, Hooton TM, Naber KG, Wullt B, Colgan R, Miller LG, et al. International clinical practice guidelines for the treatment of acute uncomplicated cystitis and pyelonephritis in women: a 2010 update by the Infectious Diseases Society of America and the European Society for Microbiology and Infectious Diseases. Clin Infect Dis. 2011;52(5):e103-20.

2. Habib G, Lancellotti P, Antunes MJ, Bongiorni MG, Casalta J-P, Del Zotti F, et al. 2015 ESC guidelines for the management of infective endocarditis: the task force for the management of infective endocarditis of the European Society of Cardiology (ESC) endorsed by: European Association for Cardio-Thoracic Surgery (EACTS), the European Association of Nuclear Medicine (EANM). European Heart J. 2015;36(44):3075-128.

3. Mermel LA, Allon M, Bouza E, Craven DE, Flynn P, O'grady NP, et al. Clinical practice guidelines for the diagnosis and management of intravascular catheterrelated infection: 2009 Update by the Infectious Diseases Society of America. Clin Infect Dis. 2009;49(1):1-45.

4. Davis BD. Mechanism of bactericidal action of aminoglycosides. Microbiol Rev. 1987;51(3):341.

5. Krause KM, Serio AW, Kane TR, Connolly LE. Aminoglycosides: an overview. Cold Spring Harbor Perspect Med. 2016;6(6):a027029.

6. Kluge RM, Standiford HC, Tatem B, Young VM, Greene WH, Schimpff SC, et al. Comparative activity of tobramycin, amikacin, and gentamicin alone and with carbenicillin against Pseudomonas aeruginosa. Antimicrobial Agents Chemotherap. 1974;6(4):442-6.

7. Sweileh WM. A prospective comparative study of gentamicin-and amikacin-induced nephrotoxicity in patients with normal baseline renal function. Fundamental Clin Pharmacol. 2009;23(4):515-20.

8. Denamur S, Tyteca D, Marchand-Brynaert J, Van Bambeke F, Tulkens PM, Courtoy PJ, et al. Role of oxidative stress in lysosomal membrane permeabilization and apoptosis induced by gentamicin, an aminoglycoside antibiotic. Free Radical Biol Med. 2011;51(9):1656-65.

9. Narayana K. An aminoglycoside antibiotic gentamycin induces oxidative stress, reduces antioxidant reserve and impairs spermatogenesis in rats. J Toxicological Sci. 2008;33(1):85-96.

10. Luthfi MJf. A Simple and Practical Method for Rat Epididymal Sperm Count (Rattus norvegicus). Biol Med Natural Product Chem. 2015;4(1):1-3.

11. Semet M, Paci M, Saïas-Magnan J, MetzlerGuillemain C, Boissier R, Lejeune $\mathrm{H}$, et al. The impact of drugs on male fertility: a review. Andrology. 2017;5(4):640-63.

12. Staff A. The Optimal Evaluation of the Infertile Male: AUA Best Practice Statement. 2010.

13. Laurence DR, Bacharach AL. Evaluation of drug activities: pharmacometrics. ASPET; 1964.

14. Carageorgiou HK, Stratakis CA, Damoulis PD, Varonos DD, Messari ID, Sideris AC, et al. Reversible plasma testosterone levels reduction after gentamicin administration and freund's adjuvant arthritis in rats. Indian $\mathrm{J}$ Physiol Pharmacol. 2005;49(4):443.

15. Kim SH, Lee IC, Baek HS, Shin IS, Moon C, Kim $\mathrm{SH}$, et al. Melatonin prevents gentamicin-induced testicular toxicity and oxidative stress in rats. Andrologia. 2014;46(9):1032-40.

16. Sanati E, Nikmanesh M. Comparative study of the effects of gentamicin, neomycin, streptomycin and ofloxacin antibiotics on sperm parameters and testis apoptosis in rats. Pakistan $\mathrm{J}$ Biological Sci. 2008;11(13):1683-9.

17. Akutsu S, Katoh M, Kawana K, Shimamura T, Fukushima Y, Matsuzaki M. Effects of intravenous infusion of amikacin. Intraperitoneal administration to rats before and in the early stage of pregnancy. Segment 1. Japanese J Antibiotics. 1982;35(8):210010.

18. Aly HA, Hassan MH. Potential testicular toxicity of gentamicin in adult rats. Biochem Biophysical Research Communications. 2018;497(1):362-7.

19. Karaman M, Budak H, Çiftci M. Amoxicillin and gentamicin antibiotics treatment adversely influence the fertility and morphology through decreasing the Dazl gene expression level and increasing the oxidative stress. Arch Physiol Biochem. 2018: 1-9.

20. Kerr J. Spontaneous degeneration of germ cells in normal rat testis: assessment of cell types and frequency during the spermatogenic cycle. Reproduction. 1992;95(3):825-30. 
21. Khaki A, Novin MG, Khaki A, Fathiazad F, Khaberi M, Hossinchi J, et al. Ultra structural study of gentamicin and ofloxacin effect on testis tissue in rats: Light and transmission electron microscopy. African J Pharm Pharmacol. 2009;3(4):105-9.

22. Nouri M, Khaki A, Fathiazar F, Rashidi MR. The protective effects of carrot seed extract on spermatogenesis and cauda epididymal sperm reserves in gentamicin treated rats. Yakhteh. 2009: 327-331.

23. Lee V, De Kretser D, Hudson B, Wang C. Variations in serum FSH, LH and testosterone levels in male rats from birth to sexual maturity. Reproduction. 1975;42(1):121-6.

24. Blanco-Rodriguez J, Martinez-Garcia C. Apoptosis precedes detachment of germ cells from the seminiferous epithelium after hormone suppression by short-term oestradiol treatment of rats. Int $\mathbf{J}$ Androl. 1998;21(2):109-15.

25. Dohle G, Smit M, Weber R. Androgens and male fertility. World J Urol. 2003;21(5):341-5.

26. Knorr D, Vanha-Perttula T, Lipsett M. Structure and function of rat testis through pubescence. Endocrinology. 1970;86(6):1298-304.

27. Khaki A, Khaki A, Iraj S, Bazi P, Imani SAM, Kachabi H. Comparative Study of Aminoglycosides (Gentamicin \& Streptomycin) and Fluoroquinolone (Ofloxacin) Antibiotics on Testis Tissue in Rats:
Light and Transmission Electron Microscopic Study. Pak J Med Sci. 2009;25(4):624-9.

28. Lenzi A, Gandini L, Lombardo F, Picardo M, Maresca V, Panfili E, et al. Polyunsaturated fatty acids of germ cell membranes, glutathione and blutathione-dependent enzyme-PHGPx: from basic to clinic. Contraception. 2002;65(4):301-4.

29. Dym M, Fawcett DW. The blood-testis barrier in the rat and the physiological compartmentation of the seminiferous epithelium. Biol Reprod. 1970;3(3):308-26.

30. Tsuji A, Tamai I. Blood-brain barrier function of Pglycoprotein. Advanced Drug Delivery Reviews. 1997;25(2-3):287-98.

31. Carlier M-B, Rollman B, Van Hoof Fi, Tulkens P. Mechanism of aminoglycoside-induced lysosomal phospholipidosis: in vitro and in vivo studies with gentamicin and amikacin. Biochem Pharmacol. 1982;31(23):3861-70.

Cite this article as: Elsawah HK, Kandiel MM, Amin AA, Mokhimar HM, El Mahmoudy AM. Gentamicin and amikacin adversely affect male infertility indicated by pharmacological, andrological and pathological evidence. Int J Basic Clin

Pharmacol 2020;9:218-25. 